\title{
CPA Theory of Superconducting Alloys with Diagonal and Off-Diagonal Disorder
}

\author{
M. Michalik AND K.I. WySOKIŃSKI \\ Institute of Physics, M. Curie-Skłodowska University \\ pl. M. Curie-Skłodowskiej 1, 20-031 Lublin, Poland
}

\begin{abstract}
The general theory of disorder in superconducting alloys was discussed. We use standard Wannier function representation and allow for diagonal and off-diagonal disorder. We generalized the coherent potential approximation in the version able to deal with off-diagonal disorder in normal systems to treat superconducting state. As an illustration we calculate the quasiparticle density of states and gap function of a $d$-wave superconductor. We show inter alia that the rate at which superconductivity disappears depends on the kind of disorder and for off-diagonal disorder it depends on the details of its realization.
\end{abstract}

PACS numbers: 71.10.--w, 71.23.--k, 74.20.--

\section{Introduction}

Discovery of high temperature and other classes of exotic superconductors [1] has stimulated the research of correlated and disordered systems. The reaction of superconductors against the impurities intentionally introduced into them is often the first indication of the non $s$-wave symmetry of the order parameter. Most of the previous work on disordered superconductors has concentrated on the studies of diluted impure systems [2-4], with few notable exceptions [5-7], where concentrated alloys have been studied with help of coherent potential approximation (CPA). In this later case only diagonal disorder has been treated. Here we apply the CPA in the version suitable to deal with off-diagonal disorder [8] to superconducting systems [9] and calculate some characteristics of the system including the gap function and the density of quasiparticle states.

We shall use the mentioned approach and study the dependence of properties of superconducting alloys on a kind and degree of disorder. Motivated by experiments [10] measuring the tunneling conductance of $\mathrm{Bi}_{2} \mathrm{Sr}_{2} \mathrm{CaCuO}_{8+\delta}$ we 
calculate the density of states and observe that off-diagonal disorder may introduce the asymmetry of the tunneling conductance notoriously observed in high-temperature superconductors. Let us note that the measurements of tunneling spectra allow determination of the density of states $N(E)$ and the dependence of the superconducting gap on temperature, magnetic field etc.

In the second section we briefly sketch CPA technique for off-diagonal disorder [8] and shortly describe our method of generalizing this technique for superconducting alloys. The numerical results for the density of states and the dependence of the superconducting gap on disorder will be presented in Sec. 3. We end up with discussion and conclusions.

\section{The model and approach}

To describe disordered systems we will use the Hubbard model formulated in the Wannier function representation

$$
H=\sum_{i j \sigma} \hat{t}_{i j} c_{i \sigma}^{+} c_{j \sigma}+\sum_{i \sigma}\left(\epsilon_{i}-\mu\right) c_{i \sigma}^{+} c_{i \sigma}+\sum_{i} U_{i} c_{i \uparrow}^{+} c_{i \uparrow} c_{i \downarrow}^{+} c_{i \downarrow}+\sum_{i, j} V_{i, j} n_{i} n_{j},
$$

in which site energies $\epsilon_{i}$ are random quantities taking on values $\epsilon_{\mathrm{A}}$ or $\epsilon_{\mathrm{B}}$ depending on whether site $i$ is occupied by an atom of $\mathrm{A}$ or $\mathrm{B}$ type. In (1) $c_{i \sigma}^{+}\left(c_{j \sigma}\right)$ is the creation (annihilation) operator of spin $\sigma$ electron at site $i, n_{i}=\sum_{\sigma} c_{i \sigma}^{+} c_{i \sigma}, \mu$ is the chemical potential and $U_{i}$ is the on-site electron-electron repulsion, while $V_{i j}$ is effective attraction leading to superconducting instability in the system. $\hat{t}_{i j}$ denote random hopping integrals taking on three values $\hat{t}_{i j}^{\mathrm{AA}}=\alpha t_{i j}, \hat{t}_{i j}^{\mathrm{AB}}=\hat{t}_{i j}^{\mathrm{BA}}=\chi t_{i j}$ and $\hat{t}_{i j}^{\mathrm{BB}}=\beta t_{i j}$ according to the type of atoms (A or B) occupying sites $i$ and $j$ respectively. $t_{i j}$ is the periodic hopping integral taking on nonzero value $-t$ for sites $i, j$ being nearest neighbors.

To calculate characteristics typical of a macroscopic system one is forced to perform averaging over disorder. For this purpose we shall use CPA in the version proposed by Blackman, Esterling, and Berk (BEB) [8] and apply it to superconductor. In the present paper we shall limit our discussion to $\mathrm{A}_{x} \mathrm{~B}_{1-x}$ alloys with $x=0.5$, being $d$-wave symmetry superconductors and systems without the Coulomb correlations $(U=0)$. The energy unit is $t$ and the chemical potential $\mu$ is assumed to lie in the middle of the clean system energy band.

Our starting point are the Bogoliubov-de Gennes [7] equations for the matrix Green $G_{i j}(\omega)$ function in Nambu-Gorkov representation

$\sum_{j}\left(\begin{array}{cc}\left(E-\epsilon_{i}+\mu\right) \delta_{i j}-t_{i j} & -\Delta_{i j} \\ -\Delta_{i j} & \left(E+\epsilon_{i}-\mu\right) \delta_{i j}+t_{i j}\end{array}\right)\left(\begin{array}{cc}G_{j l}^{11}(E) & G_{j l}^{12}(E) \\ G_{j l}^{21}(E) & G_{j l}^{22}(E)\end{array}\right)=\delta_{i l}$

where $\Delta_{i j}=V_{i j}\left\langle c_{i \uparrow}^{+} c_{j \downarrow}^{+}\right\rangle$. Denoting

$$
W_{i l}=\left(\begin{array}{cc}
t_{i l} & \Delta_{i l} \\
\Delta_{i l} & -t_{i l}
\end{array}\right)
$$


and

$$
g_{i}^{-1}(E)=\left(\begin{array}{cc}
E-\epsilon_{i} & 0 \\
0 & E+\epsilon_{i}
\end{array}\right),
$$

we rewrite the equation of motion as

$$
\begin{aligned}
& \left(\begin{array}{ll}
G_{11}(E) & G_{12}(E) \\
G_{21}(E) & G_{22}(E)
\end{array}\right)_{i j}=\left(\begin{array}{cc}
\frac{1}{E-\epsilon_{i}} & 0 \\
0 & \frac{1}{E+\epsilon_{i}}
\end{array}\right) \delta_{i j}+\left(\begin{array}{cc}
\frac{1}{E-\epsilon_{i}} & 0 \\
0 & \frac{1}{E+\epsilon_{i}}
\end{array}\right) \\
& \times \sum_{l \neq i}\left(\begin{array}{ll}
W_{11} & W_{12} \\
W_{21} & W_{22}
\end{array}\right)_{i l}\left(\begin{array}{ll}
G_{11}(E) & G_{12}(E) \\
G_{21}(E) & G_{22}(E)
\end{array}\right)_{l j}
\end{aligned}
$$

or in the compact notation

$$
G_{i j}(E)=g_{i}(E) \delta_{i j}+g_{i}(E) \sum_{i \neq l} W_{i l} G_{l j}(E) .
$$

Now the idea is to project out the disorder by using operators $x_{i}\left(y_{i}\right)$ taking on value 1 if site $i$ is occupied by an $\mathrm{A}(\mathrm{B})$-type atom and 0 otherwise. The operators (projectors) have the following properties: $x_{i}+y_{i}=1$, $x_{i} y_{i}=y_{i} x_{i}=0, x_{i} x_{i}=x_{i}, y_{i} y_{i}=y_{i}$. To perform the projection one multiplies Eq. (2) from the left and right by all possible combinations of projectors and makes use of their properties. In the normal state this leads to four equations [8], which can be easily solved numerically. For superconducting alloy one gets in total sixteen equations. The first of them appropriate for two atoms both of type A at sites $i, j$ and for matrix element $G_{i j}^{11}$ reads

$$
\begin{aligned}
& x_{i} G_{i j}^{11} x_{j}=\frac{x_{i}}{E-\epsilon_{\mathrm{A}}} \delta_{i j}+\frac{x_{i}}{E-\epsilon_{\mathrm{A}}} \sum_{k \neq i}\left[W_{i k}^{11}\left(x_{k}+y_{k}\right) G_{k j}^{11}\right. \\
& \left.+W_{i k}^{12}\left(x_{k}+y_{k}\right) G_{k j}^{21}\right] x_{j} .
\end{aligned}
$$

Now both hopping integrals and pairing potentials take on the fixed (for a given pair of atoms at sites $i, j$ ) and non-random value. To see this let us write them explicitly: $\hat{t}_{k i}=\left(x_{k} \alpha x_{i}+y_{k} \beta y_{i}+x_{k} \chi y_{i}+y_{k} \chi x_{i}\right) t_{k i}, \quad \Delta_{k i}=$ $\left(x_{k} \delta_{\mathrm{AA}} x_{i}+y_{k} \delta_{\mathrm{BB}} y_{i}+x_{k} \delta_{\mathrm{AB}} y_{i}+y_{k} \delta_{\mathrm{BA}} x_{i}\right) \Delta_{k i}^{0} . \quad \Delta_{k i}^{0}$ denotes the pairing potential of arbitrary symmetry for clean system, while $\delta_{\mathrm{AA}}, \delta_{\mathrm{BB}}, \delta_{\mathrm{AB}}, \delta_{\mathrm{BA}}$ are corrections to $\Delta_{k i}$.

We have started with a 2 by 2 equation for the matrix Green function and after averaging end up with a 4 by 4 matrix equation for the averaged matrix elements. They are supplemented by 4 conditions for $\delta_{\mathrm{AA}} \Delta_{k i}^{0}$ etc., which are calculated from the corresponding matrix element of the Green function.

From the single impurity calculations we know that there are no self-energy corrections to the $d$-wave order parameter induced by isotropic impurity scattering [2]. One can thus safely assume [7] that this is also the case in the present situation 
and write down equation for the order parameter as [11]:

$$
\sum_{k}\left(\begin{array}{cc}
\mathcal{G}_{k}^{\mathrm{BEB}} & \Delta_{k} \\
\Delta_{k} & \left(\mathcal{G}_{k}^{\mathrm{BEB}}\right)^{*}
\end{array}\right)\left(\begin{array}{ll}
\mathcal{G}_{11} & \mathcal{G}_{12} \\
\mathcal{G}_{21} & \mathcal{G}_{22}
\end{array}\right)=\left(\begin{array}{ll}
1 & 0 \\
0 & 1
\end{array}\right)
$$

This means that it is the normal state of an alloy which is subject of change by disorder. In the Abrikosov-Gorkov approach to $d$-wave impure superconductor [2] this corresponds to modifications of the frequency only. We calculate functions $\mathcal{G}_{k}^{\mathrm{BEB}}$ from CPA equations in the BEB formulation for the normal state, and then $\mathcal{G}_{11}$ from Eq. (3). It reads

$$
\mathcal{G}_{11}=\sum_{k} \frac{\left(\mathcal{G}_{k}^{\mathrm{BEB}}\right)^{-1}}{\left|\mathcal{G}_{k}^{\mathrm{BEB}}\right|^{-2}+\Delta_{k}^{2}}
$$

Quasiparticle density of states at energy $E$ is given by the imaginary part of $\mathcal{G}_{11}(E)$ :

$$
D(E)=-\frac{1}{\pi} \operatorname{Im} \mathcal{G}_{11}(E)
$$

This quantity is directly proportional to the tunnel conductance $\mathrm{d} I / \mathrm{d} V$ and gives information on the superconducting gap [10].

\section{Results}

We have solved the CPA equations for the normal state Green function $\mathcal{G}_{k}^{\mathrm{BEB}}$ and calculated the order parameter and the density of quasiparticle states which, as already mentioned, can be directly measured via tunneling experiments. Disorder dependence of the density of states is shown in Fig. 1a,b. Figure 1a shows the changes of the density of states with diagonal disorder only. In this case disorder is measured by $\delta=\epsilon_{\mathrm{A}}-\epsilon_{\mathrm{B}}$ in units of $t$. One can see that the main effect is
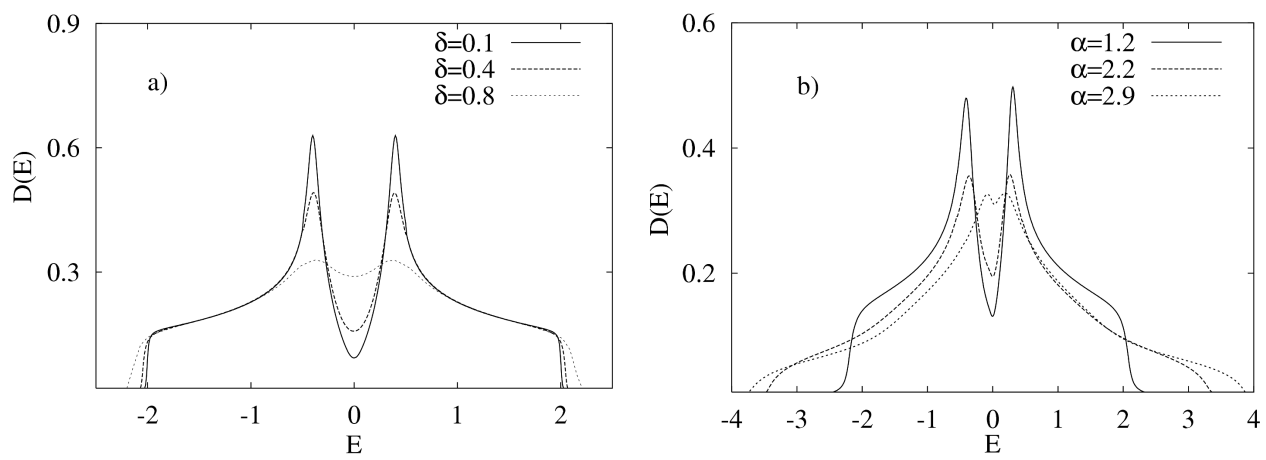

Fig. 1. Density of states of an $\mathrm{A}_{x} \mathrm{~B}_{1-x}$ alloy with varying diagonal disorder $\delta$, and no off-diagonal disorder $\alpha=\beta=\chi=1$ (a) and with off-diagonal disorder only corresponding to $\chi=\beta=1$, and $\alpha=1.2,2.2,2.9, \delta=0$ (b). 


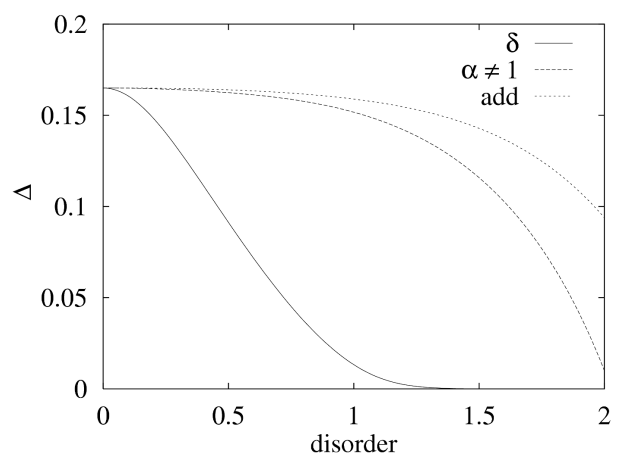

Fig. 2. Changes of $\Delta$ with disorder. The curve marked $\delta$ corresponds to diagonal disorder only when disorder $=\delta$, curve marked $\alpha \neq 1$ corresponds to off-diagonal disorder with $\chi=\beta=1$ and disorder $=\alpha-1$ while curve marked $a d d$ to additive one with $\chi=(\alpha+\beta) / 2$.

connected with the appearance of states inside the $d$-wave gap and its subsequent closing. For off-diagonal disorder the changes are more complex. If we keep the parameters $\beta$ and $\chi$ describing off-diagonal disorder at their clean metal value 1 and change only $\alpha$ (as indicated) we observe appearance of the asymmetry in the $\mathrm{V}$-shaped gap (both in the heights of the peaks and the slopes near zero) and the broadening of the density of states.

In Fig. 2 we show the dependence of the zero temperature gap on the disorder. The curve marked $\delta$ corresponds to Fig. 1a and shows $\Delta$ as function of diagonal disorder $\delta$. Other curves correspond to an alloy with purely off-diagonal disorder $(\delta=0)$ which for the curve marked $\alpha \neq 1$ changes as disorder $=\alpha-1$. The curve marked add corresponds to the system with additive disorder i.e. $\chi=(\alpha+\beta) / 2$. Here $\chi=1$ and the difference $|\alpha-\beta|$ can be taken as a measure of disorder. The most interesting point is to note that the rate at which off-diagonal disorder suppresses $\Delta$ and thus superconductivity in disordered $d$-wave superconducting alloy depends on the details how the disorder is introduced.

\section{Summary and conclusions}

In this paper we have generalized the CPA formalism in the formulation of Blackman, Esterling, and Berk [8] to the case of $d$-wave superconducting alloys and calculated the gap function and the quasiparticle density of states. Density of states of an 50:50 alloy with diagonal disorder only and for half-filled band is symmetric while off-diagonal disorder introduces important asymmetries.

We have noticed that the way the gap changes with disorder depends on the kind of disorder in the system. Typically the diagonal disorder suppresses the $d$-wave gap more rapidly. As there are many ways of introducing the off-diagonal disorder into the system i.e. different ways to make the numbers $\alpha, \beta, \chi$ differ 
from 1 we have calculated energy gap for two cases and found marked differences in its behavior. The detailed study of the properties of superconducting alloys with off-diagonal disorder will be presented elsewhere [12].

\section{Acknowledgments}

This work was partially supported by the State Committee for Scientific Research grant No. 2P03B 06225.

\section{References}

[1] J.G. Bednorz, K.A. Muller, Z. Phys. B 64, 89 (1986); B. Brandow, Phys. Rep. 296, 1 (1998).

[2] L.P. Gorkov, P.A. Kalugin, Pis'ma Zh. Eksp. Theor. Fiz. 41, 208 (1985) [JETP Lett. 41, 253 (1985)].

[3] R. Fehrenbach, Phys. Rev. Lett. 77, 1849 (1996).

[4] D.F. Agterberg, Phys. Rev. B 60, R749 (1999).

[5] K.I. Wysokiński, A.L. Kuzemsky, J. Low Temp. Phys. 52, 81 (1983).

[6] A.A. Nersesyan, A.M. Tsvelik, F. Wenger, Phys. Rev. Lett. 72, 2628 (1994).

[7] A.M. Martin, G. Litak, B.L. Györffy, J.F. Annett, K.I. Wysokiński, Phys. Rev. B 60, 7523 (1999).

[8] J.A. Blackman, D.M. Esterling, N.F. Berk, Phys. Rev. B 4, 2412 (1971).

[9] M. Michalik, K.I. Wysokiński, Phys. Status Solidi B 236, 372 (2003).

[10] Ch. Renner, B. Revaz, J.Y. Genoud, K. Kadowaki, O. Fischer, Phys. Rev. Lett. 80, 149 (1998).

[11] A. Romano, C. Noce, R. Micnas, Phys. Rev. B 55, 12640 (1997).

[12] M. Michalik, K.I. Wysokiński, in preparation. 